A N N A L E S Annales de Bretagne et des Pays de l'Ouest

\title{
Seigneurs résidants et notabilité dans la vallée du Saint-Laurent (XVIIe-XIXe siècle)
}

\section{Benoît Grenier}

\section{OpenEdition}

Journals

\section{Édition électronique}

URL : http://journals.openedition.org/abpo/1415

DOI : $10.4000 / a b p o .1415$

ISBN : 978-2-7535-1490-4

ISSN : 2108-6443

Éditeur

Presses universitaires de Rennes

\section{Édition imprimée}

Date de publication : 20 juillet 2003

Pagination : $59-75$

ISBN : 978-2-86847-874-0

ISSN : 0399-0826

Référence électronique

Benoît Grenier, "Seigneurs résidants et notabilité dans la vallée du Saint-Laurent (XVIIe-XIXe siècle) », Annales de Bretagne et des Pays de l'Ouest [En ligne], 110-2 | 2003, mis en ligne le 20 juillet 2005,

consulté le 20 avril 2019. URL : http://journals.openedition.org/abpo/1415 ; DOI : 10.4000/abpo.1415 


\title{
Seigneurs résidants et notabilité dans la vallée du Saint-Laurent $\left(\mathrm{XVII}^{\mathrm{e}} \text {-XIX }{ }^{\mathrm{e}} \text { siècle }\right)^{1}$
}

\author{
Benoît GRENIER \\ Doctorant en histoire \\ Université Rennes 2 Haute-Bretagne - Université Laval, Québec
}

À compter de la première moitié du XvII ${ }^{e}$ siècle, la France instaura une colonie de peuplement dans le territoire de la vallée du Saint-Laurent ${ }^{2}$. Dès lors, c'est par l'intermédiaire du système seigneurial que tout l'espace fut découpé, puis concédé à des seigneurs qui y installèrent des colons venus d'outre-Atlantique. La Conquête britannique, scellée par le traité de Paris (1763), fit passer les descendants des immigrants français sous le contrôle d'une nouvelle métropole, anglaise, mais le conquérant préserva le régime seigneurial, qui ne fut aboli qu'en 1854 à l'issu de débats parlementaires.

Pendant les deux siècles et demi au cours desquels la vallée du SaintLaurent vécut sous le système seigneurial, les censitaires côtoyèrent assez peu leurs seigneurs. La présence permanente des seigneurs sur leurs terres constituait alors une réalité plutôt inhabituelle, contrairement à ce qu'affirment la croyance populaire et une historiographie ancienne ${ }^{3}$. Alors qu'en France, au cours de l'époque moderne, les seigneurs absentéistes parais-

1. Le présent texte est issu de nos recherches doctorales sur les seigneurs résidants de la vallée du Saint-Laurent (Québec) effectuées en co-tutelle sous la direction de Madame Annie Antoine à l'Université Rennes 2 Haute-Bretagne, (France) et de Monsieur Alain Laberge à l'Université Laval (Québec, Canada).

2. Nous utilisons l'expression "vallée du Saint-Laurent " qui est la plus appropriée pour désigner ce territoire qui, entre le XVII ${ }^{\mathrm{e}}$ et le XIX ${ }^{\mathrm{e}}$ siècle, connut plusieurs appellations au gré des changements de systèmes politiques (Nouvelle-France, Canada, Québec, BasCanada, Canada-Est puis à nouveau Québec).

3. Dès le XIXe siècle, les historiens québécois, dans un contexte idéologique visant à glorifier les " origines de la Nation ", se sont employés à présenter les seigneurs comme des colonisateurs et le régime seigneurial comme un système dont les censitaires canadiens auraient bénéficié. Cette historiographie traditionnelle a, jusque dans les années 1960-1970, influencé la recherche historique sur la période de la Nouvelle-France. Consulter notamment : JAUMaIN, Serge, SANFILIPPO, Matteo, "Le régime seigneurial en Nouvelle-France : Un débat historiographique ", The Register, vol. 5, n 2, 1980, p. 226-247. 
sent devenir de plus en plus nombreux ${ }^{4}$, les seigneurs canadiens, en pays de colonisation, sont confrontés, à cette même époque, à la difficulté de prendre résidence dans des seigneuries en "bois debout ". Au fil du temps, les seigneuries se peuplèrent, mais les seigneurs, retenus alors à la ville par d'autres fonctions (militaires, administratives ou politiques), souvent désintéressés du développement de leurs fiefs, furent rarement intégrés à la société rurale ${ }^{5}$. Cet absentéisme seigneurial nous a incité à nous interroger sur la minorité qui, dans la vallée du Saint-Laurent, réside en permanence et de manière intergénérationnelle sur la terre seigneuriale.

La présence de la famille seigneuriale dans certaines communautés rurales du monde laurentien conduit à l'étude des rapports sociaux dans ces seigneuries. Dans cette optique, la question de la notabilité s'est avérée fondamentale, tant en raison du statut prestigieux de la famille seigneuriale qu'en regard des rapports de celle-ci avec les autres notables de la collectivité à laquelle elle appartient. Outre ces liens entre seigneurs et notables locaux, il importe également de nous interroger à propos de la notabilité des seigneurs eux-mêmes. Nous verrons que les particularismes de la colonie laurentienne soulèvent, en certains cas, une remise en question du statut du seigneur résidant.

Le présent texte constitue une réflexion sur la notabilité et la seigneurie dans la vallée du Saint-Laurent pour la durée entière du régime seigneurial (1626-1854) ${ }^{6}$. Afin d'illustrer les spécificités de la notabilité dans les seigneuries laurentiennes habitées par la famille seigneuriale, il faut d'abord présenter les caractéristiques de la notabilité qui se développe et se transforme au cours de la période étudiée. Ensuite, nous aborderons le contexte seigneurial dans le territoire de peuplement qu'est la vallée du Saint-Laurent, en mettant l'accent sur la mobilité sociale qui introduit des individus d'humbles origines au sein des propriétaires de seigneuries. Finalement, nous terminerons par un questionnement portant sur la difficile intégration à la notabilité de certaines familles seigneuriales résidantes auxquelles nous nous intéresserons plus spécifiquement.

4. Jean Gallet, dans une synthèse d'histoire sur la seigneurie en France pour les deux derniers siècles de l'Ancien Régime abordant la question de l'évolution de la résidence seigneuriale pendant cette période, constate l'accroissement de l'absentéisme des seigneurs. Il évoque certains particularismes régionaux, mais peint un portrait général où les seigneurs, urbanisés, se sont éloignés de leurs terres et de leurs paysans, ne conservant souvent à la campagne qu'une résidence secondaire : GALLET, Jean, Seigneurs et paysans en France, 1600-1793, Rennes, Éditions Ouest-France, 1999, p. 198-200.

5 . Selon nos travaux en cours sur cette question, il semble qu'un seigneur sur quatre ait été résidant dans la vallée du Saint-Laurent entre le XVII ${ }^{\mathrm{e}}$ et le XIX ${ }^{\mathrm{e}}$ siècle. La présence des seigneurs sur leurs terre s'accroît au cours de cette période et les résidants comptent pour près de $40 \%$ des propriétaires de seigneuries à la veille de l'abolition du régime seigneurial (1854).

6. L'année 1626 marque l'octroi des premiers titres seigneuriaux en Nouvelle-France (sur les hauteurs de la future ville de Québec), tandis que l'année 1854 est celle de l'abolition du régime seigneurial par l'Acte seigneurial du parlement du Canada-Uni. 


\section{Les notables d'une société rurale " simplifiée "}

Malgré une indéniable transformation entre le XVII et le $\mathrm{XIX}^{\mathrm{e}}$ siècle, la vallée du Saint-Laurent demeure une colonie et une société largement façonnée par le processus du peuplement. De ce fait, elle ne possédait pas l'ensemble des structures et des institutions des sociétés rurales européennes plus anciennes. "L'éventail social est moins ouvert qu'en France ", écrit Jacques Mathieu à propos de la structuration sociale de la NouvelleFrance $^{7}$. Il apparaît donc pertinent de s'interroger sur la notabilité dans cette société qui apparaît comme "simplifiée " et ce tout particulièrement dans le monde rural.

Traversant le régime français et la presque totalité du régime britannique, aboli à peine une décennie avant la Confédération canadienne, le régime seigneurial constitua le cadre de vie de plus d'une catégorie de notables. En résumant la transformation de l'élite locale de la seigneurie de Sainte-Anne-de-la-Pérade ${ }^{8}$, entre le XVII ${ }^{\mathrm{e}}$ et le XIX ${ }^{\mathrm{e}}$ siècle, l'historien Colin Coates décrit une situation qui peut être généralisée à l'ensemble du territoire seigneurial de la vallée du Saint-Laurent :

"The composition of the elite changed over time. Fully involved in defining local issue during the French regime, seigneurs has become more distant from those concerns by the early nineteenth century. Meanwhile, the local clergy broadened their influence, as did militia captains. Over time, the process of economic differentiation in the countryside created a petty bourgeoisie of merchants and larger-scale farmers. The introduction of parliamentary elections in 1792 provided a potential public forum for this non-noble lay elite ${ }^{9}$."

Cette transformation de la notabilité s'explique par le développement du territoire laurentien et l'accroissement de sa population après les décennies d'implantation, mais aussi par l'avènement du régime britannique qui, sans remettre en cause les fondements de la société mise en place par la France dans la vallée du Saint-Laurent, allait offrir de nouvelles voies permettant d'entrer en notabilité.

Les institutions qui encadrent la vie rurale sous le régime français, en raison de la faiblesse et de la dispersion de la population, n'ont pas repro-

7. MathiEu, Jacques, La Nouvelle-France. Les Français en Amérique du Nord XVI ${ }^{e}$-XVIII siècle, Paris/Québec, Belin/Presses de l'Université Laval, 1991, p. 191 (nouvelle édition en 2001).

8. Située dans le gouvernement de Trois-Rivières, la seigneurie de Sainte-Anne-de-laPérade était possédée par les Tarieu de Lanaudière, famille noble.

9. MACMILlan COATES, Colin, The Metamorphoses of Landscape and Community in Early Quebec, Montréal/Kingston, McGill/Queen's University Press, 2000, p. 75-76. Notre traduction : "La composition de l'élite s'est transformée avec le temps. Complètement impliquée dans l'organisation des affaires locales durant le régime français, les seigneurs sont devenus plus distants de ces réalités au début du XIXe siècle. Pendant cette période, le clergé local a renforcé son influence, tout comme les capitaines de milice. Au fil du temps, le processus de différentiation économique dans les campagnes a créé une petite bourgeoisie de marchands et d'agriculteurs prospères. L'introduction des élections parlementaires, en 1792, procura une tribune publique potentielle à cette élite lä̈que roturière." 
duit à l'identique les structures sociales qui prévalaient généralement en France. Conséquemment, l'accès à la notabilité s'en trouve limité. Seigneurie, paroisse, milice et, au fil du temps, bourgs et villages, constituent pratiquement les seules institutions d'encadrement de la vie rurale que connaissent les habitants ${ }^{10}$ et qui ouvrent les portes de la notabilité. À ces institutions, il faut ajouter la famille et le voisinage qui constituent les tous premiers cadres communautaires de la Nouvelle-France. La famille, institution fondamentale en France, joue en Nouvelle-France un rôle accentué et central en raison de l'isolement des habitations et de l'absence de vie collective organisée, surtout au XVII ${ }^{\mathrm{e}}$ siècle. Dans cette société rurale simplifiée, les notables que côtoient les seigneurs résidants composent une élite restreinte.

La paroisse, et plus particulièrement son titulaire, le curé, constituait un pouvoir local dont l'influence risquait de concurrencer celui des seigneurs, notamment lorsque ceux-ci ne résidaient pas dans la seigneurie. Toujours au sein de l'institution paroissiale, les marguilliers, élus annuellement pour administrer les biens de la paroisse, comptent aussi parmi les notables de la localité ${ }^{11}$. L'institution de la milice, regroupée en fonction des limites paroissiales, et non seigneuriales, représentait un autre pôle d'autorité au sein de la communauté locale. Les capitaines de milice, élus parmi les principaux habitants de la paroisse, étaient revêtus d'une fonction qui n'était pas seulement honorifique; la participation des hommes à cette institution était obligatoire et les périodes de guerre furent nombreuses aux XVII ${ }^{\mathrm{e}}$ et XVIII ${ }^{\mathrm{e}}$ siècles ${ }^{12}$. Sous le régime français, la seigneurie est aussi créatrice de notables locaux, du moins dans les régions où la population est implantée : meunier, procureur fiscal, notaire et juge seigneurial sont dotés d'une certaine supériorité sociale ${ }^{13}$.

Face au curé et au capitaine de milice auxquels, avec le temps, s'ajoutèrent de nouvelles élites locales, on présente habituellement le seigneur comme le premier notable de la communauté, d'autant plus qu'il faisait aussi parfois fonction d'officier de milice, accroissant par le fait même son pouvoir sur la communauté locale ${ }^{14}$. En effet, il n'est pas rare qu'un seigneur détienne d'autres fonctions qu'il exerçait parfois avant de d'avoir acquis une terre. Ces occupations ne sont pas toujours liées au territoire seigneurial, mais elles permettent de comprendre la concentration des fonctions qui font un notable entre les mains du petit groupe auquel appartiennent de nombreux seigneurs. À la fin du XVII ${ }^{\mathrm{e}}$ siècle, Charles Aubert de la Chesnaye

10. Dans la vallée du Saint-Laurent, on utilise, dès le Régime français, le terme « habitant " en lieu et place de celui de paysan; le mot " habitant " a une connotation positive qui témoigne de la propriété de la terre. MATHIEU, Jacques, La Nouvelle-France..., op. cit., p. 47.

11. Mathieu, Jacques, La Nouvelle-France..., op. cit., p. 194.

12. OuELLET, Fernand, " Officiers de milice et structure sociale au Québec (1660-1815) ", Histoire Sociale, vol. 12, n 23 (1979), p. 37-65.

13. Mathieu, Jacques, La Nouvelle-France..., op. cit., p. 194-195.

14. OuEllet, Fernand, "Officiers de milice... ", art. cit., p. 44. 
(1632-1702), marchand, acquiert plusieurs seigneuries; bien qu'anobli, il continue le commerce et le négoce. Un siècle plus tard, Antoine Juchereau Duchesnay (1740-1806), seigneur de Beauport, se fait élire député, puis devient conseiller législatif; James Cuthbert (1719-1798), officier britannique originaire d'Écosse, achète des seigneuries après la Conquête de la NouvelleFrance, notamment celle de Berthier où il réside, mais exerce parallèlement diverses fonctions : marchand, juge de paix, conseiller législatif; en 1720, l'abbé Louis Lepage de Sainte-Claire (1690-1762), lui-même fils du seigneur résidant de Rimouski, acquiert la seigneurie de Terrebonne (au nord de Montréal) tout en étant le desservant religieux de l'endroit ${ }^{15}$. Des seigneurs sont à la fois capitaines de milice, ou encore, à la fin de la période seigneuriale, maires, ce qui leur procure une double notabilité locale. Ainsi, on comprend que le cumul de diverses fonctions honorifiques par des seigneurs, surtout s'il sont résidants, vient affermir leur prestige.

La récupération et la préservation par le conquérant britannique de plusieurs institutions de l'époque française, telles la milice, la paroisse et la seigneurie, firent en sorte que certaines catégories de notables demeurèrent inchangées sous ces deux régimes. Malgré le changement de métropole, on peut davantage parler de continuité que de rupture; la société rurale, dans les seigneuries laurentiennes, demeure francophone, catholique et encadrée par les mêmes institutions. Ainsi, le seigneur, le prêtre et le capitaine de milice continuent à exercer leurs fonctions respectives au sein des communautés rurales pendant toute la période qui nous intéresse. La notabilité rurale connaît cependant certaines évolutions à compter des dernières décennies du XVIII ${ }^{\mathrm{e}}$ siècle. Ainsi, l'importance des professionnels s'accroît avec l'avènement du régime britannique; médecins, notaires, avocats et arpenteurs ${ }^{16}$ occupent de plus en plus de place au sein de la notabilité rurale. L'une des tribunes qui leur permit de se distinguer progressivement et d'occuper l'avant-plan dans la collectivité fut l'avènement du parlementarisme en $1791^{17}$. Si les seigneurs occupèrent d'abord une place indéniable au sein de la classe politique, les professionnels, pendant la première moitié du XIX ${ }^{e}$ siècle, composent la majeure partie des élus au Parlement du Bas-Canada ${ }^{18}$.

15. LABERGE, Alain, MathieU, Jacques (dir.), avec la collaboration de François CANTARA, "Répertoire seigneurial de la vallée du Saint-Laurent, XVII ${ }^{\mathrm{e}}$-XIX ${ }^{\mathrm{e}}$ siècle ", (non publié), département d'histoire, Université Laval (Québec).

16. Si les notaires étaient présents dès le régime français et continuèrent à exercer jusqu'à nos jours, constituant les premiers professionnels, les avocats "qui ont la réputation d'embrouiller les affaires " étaient interdits par la monarchie dans la colonie sous le régime français : MATHIEU, Jacques, La Nouvelle-France..., op. cit., p. 98.

17. L'Acte constitutionnel de 1791 créait deux territoires distincts (Bas-Canada et HautCanada correspondant aux actuelles provinces du Québec et de l'Ontario) et instituait une chambre d'assemblée pour chacun. Les premières élections se déroulèrent en 1792. (VAugeols, Denis, Québec 1792 : Les acteurs, les institutions et les frontières, Montréal, Fides, 1992.)

18. Ibid., p. 127; OuELlET, Fernand, Le Bas-Canada 1791-1840 : Changements structuraux et crise, Ottawa, Éditions de l'Université d'Ottawa, 1976, 541 p. 
La création des corporations municipales, au milieu du XIX ${ }^{\mathrm{e}}$ siècle, entraîne l'apparition des fonctions de maire et d'échevin, elles aussi marquées du sceau de la notabilité. Au sein des communautés, de nouvelles fonctions de pouvoir et de prestige s'affirment. Dans les paroisses rurales, après la période colonisatrice, qui tend à uniformiser les statuts sociaux, la diversité se manifeste et divers acteurs viennent alimenter la composition de l'élite locale : maître d'école, artisans, marchands ruraux, etc. Au sein même de la paysannerie, la diversité socio-économique, doit aussi être prise en compte. D'importantes familles d'agriculteurs prospères se distinguent des habitants ${ }^{19}$. Les " vieilles " familles qui, au XVII ${ }^{\mathrm{e}}$ siècle, furent des pionnières d'une communauté occupent souvent les meilleures terres, celles situées au premier rang, près du fleuve ${ }^{20}$. Ces familles bien enracinées dans la paroisse et la seigneurie parviennent souvent à un seuil de respectabilité non négligeable, à des fonctions de marguilliers ou d'échevins par exemple. Ce portrait, très sommaire, de la notabilité de la vallée du Saint-Laurent nous permet de comprendre qu'au terme du régime seigneurial (1854), la société rurale en place s'est transformée et que les types de notables sont plus nombreux qu'au XvII ${ }^{\mathrm{e}}$ siècle.

Cette complexification de la structure sociale ainsi que l'émergence de nouveaux notables plaça les seigneurs face à de multiples concurrents potentiels au sein du monde rural. Néanmoins, le seigneur put se cramponner à son statut, continuer à percevoir les rentes et autres droits seigneuriaux, tout en bénéficiant, de son vivant et après sa mort, de la préséance à l'église paroissiale. De plus, l'aura seigneuriale semble faciliter l'accès des membres de la famille seigneuriale à d'autres fonctions réservées à la notabilité. Ainsi, même après l'abolition du régime seigneurial, des membres des familles seigneuriales résidantes deviendront maires dans les territoires correspondant à leurs anciennes seigneuries ${ }^{21}$. Le groupe seigneurial de la vallée du Saint-Laurent n'est cependant pas homogène et la partie qui suit vise à démontrer l'influence du contexte colonial sur la mobilité sociale et la construction du groupe des seigneurs résidants.

19. Il s'agit d'une question récente dans l'historiographie québécoise puisque l'idée de l'uniformité parmi les habitants a longtemps persisté. Sur la question de la hiérarchisation économique de la paysannerie dans le Québec préindustriel : DESSUREAULT, Christian, "Les fondements de la hiérarchie sociale au sein de la paysannerie : le cas de SaintHyacinthe, 1760-1815 ", thèse de doctorat, Montréal, Université de Montréal, 1985; " L'égalitarisme paysan dans l'ancienne société rurale de la vallée du Saint-Laurent : Éléments pour une réinterprétation ", Revue d'histoire de l'Amérique française, vol. 40, n 3 (hiver 1987).

20. LABERGE, Alain (dir.), Histoire de la Côte-du-Sud, Québec, IQRC, 1993.

21. À la veille de l'abolition du régime seigneurial et après celle-ci, les exemples de seigneurs-maires se rencontrent à plusieurs occasions dans la vallée du Saint-Laurent. À Saint-Hyacinthe ou à Trois-Pistoles, la famille seigneuriale est intimement liée au nouveau pouvoir municipal: Société d'histoire régionale de Saint-Hyacinthe, Saint-Hyacinthe 1748-1998, Sillery, Septentrion, 1998; RIoux, Emmanuel, Histoire de Trois-Pistoles, 16971997, Trois-Pistoles, Centre d'édition Les Basques, 1997. 


\section{La seigneurie dans la vallée du Saint-Laurent : peuplement et notabilité seigneuriale}

Pour bien saisir les caractéristiques de la notabilité seigneuriale dans la vallée du Saint-Laurent, il est nécessaire de rappeler brièvement les particularismes de la seigneurie dans le peuplement du territoire, afin d'en dégager les conséquences sur la mobilité sociale et l'émergence d'une notabilité seigneuriale d'origine modeste.

Pendant le régime français, plus de trois cents seigneuries furent concédées dans la colonie canadienne ${ }^{22}$ : $75 \%$ à des seigneurs laïcs et $25 \%$ au clergé $^{23}$. Le clergé fut doté de très grandes seigneuries comptant parmi les premières peuplées, parce que bien situées (notamment Beaupré, l'île d'Orléans, Montréal, l'île Jésus...). De leur côté, les seigneurs laïcs se caractérisent en grande majorité par leur absentéisme. Dans le cadre de nos recherches, nous avons pu déterminer le taux de résidence des seigneurs de la vallée du Saint-Laurent. Il s'avère que les résidants constituent toujours une minorité parmi les quelque 1227 seigneurs laïcs répertoriés au cours de la durée du régime seigneurial ${ }^{24}$. Le plus haut taux de résidence (près de $40 \%$ ) est noté à la veille de l'abolition du régime seigneurial, vers $1850^{25}$.

Parmi l'ensemble des familles seigneuriales résidantes, nous nous sommes intéressé de manière plus spécifique à celles qui résident dans la longue durée et dans un cadre intergénérationnel. Nous en avons retenu dix, réparties dans les différentes régions de la vallée du Saint-Laurent, à savoir les gouvernements de Québec, Montréal et Trois-Rivières. Certaines seigneuries, telles Beauport ou Boucherville occupent une position privilégiée, près des villes et réseaux commerciaux et sociaux. D'autres, au contraire, telles L'Isle-Verte et Trois-Pistoles, sont fort éloignées, constituant les limites orientales du peuplement laurentien ${ }^{26}$.

Dans la vallée du Saint-Laurent, pas plus qu'en France, la propriété seigneuriale n'est synonyme de noblesse. Ainsi, parmi les seigneurs, on retrouve des nobles aussi bien que des roturiers. Le sous-groupe auquel nous nous intéressons se compose de six familles roturières et de quatre

22. Pour une cartographie du territoire seigneurial à son apogée, quelques précieux ouvrages : Louise DECHENE et Richard C. HARRIS (dir.), Atlas historique du Canada, vol. 1. Des origines à 1800, Montréal, Presses de l'Université de Montréal, 1987, planche 51. CourVILle, Serge, LabrecQue, Serge, Seigneuries et fiefs du Québec : nomenclature et cartographie, Sainte-Foy, CÉLAT et Québec, Commission de toponymie, 1988, 202 p.

23. WALLOT, Jean-Pierre, "Le Régime seigneurial et son abolition au Canada ", The Canadian Historical Review, vol. 50, n 1 (décembre 1969), p. 370.

24. Parmi ces 1227 propriétaires seigneuriaux, environ $25 \%$ possèdent plus d'une seigneurie sur le 226 répertoriées (laïques).

25. Les données relatives aux taux de résidence seigneuriale seront fournies dans notre thèse (en cours de rédaction).

26. Dans le cadre de nos travaux, dix seigneuries ont été étudiées : Beauport, Grondines, Rivière-du-Sud, L'Isle-Verte et Trois-Pistoles (Gouvernement de Québec); Baie-SaintAntoine et Saint-François-du-Lac (Gouvernement de Trois-Rivières); Lavaltrie, Boucherville et Saint-Hyacinthe (Gouvernement de Montréal). 
familles nobles, dont trois anoblies au Canada et issues de milieux modestes. Lorsqu'on observe la totalité des seigneurs résidants, les roturiers, principalement les familles seigneuriales les plus modestes, résident beaucoup plus fréquemment dans leurs fiefs que les nobles qui exercent habituellement d'autres fonctions qui les retiennent à la ville. Cette présence de nombreux individus très humbles parmi les propriétaires seigneuriaux laurentiens ${ }^{27}$, particulièrement au sein des résidants, à une époque où, en France, de telles ascensions sociales apparaissent plus difficiles, mérite notre attention.

Dans la France d'Ancien Régime, Jean-Marie Constant explique que, « au $\mathrm{XVII}^{\mathrm{e}}$ siècle [...], il ne fut plus possible à ce monde paysan d'entrer directement en noblesse. Désormais, il leur fallait suivre un cursus qui les obligeait à devenir des hommes de loi ou à passer à la ville où ils devaient franchir toutes les étapes qui conduisaient à la notabilité ${ }^{28}$ ". Au même moment, dans la vallée du Saint-Laurent, des paysans français émigrés pouvaient encore espérer une étonnante ascension sociale.

Le contexte colonial qui prévaut dans la vallée du Saint-Laurent a joué en faveur de quelques-uns parmi les plus humbles, principalement au $\mathrm{XVII}^{\mathrm{e}}$ siècle. L'accès à la terre d'individus dépourvus en France de tous biens devient possible dans la colonie. Louise Dechêne considérait comme un phénomène capital cette mobilité sociale individuelle en Nouvelle-France : "C'est au dernier palier de l'échelle sociale que la promotion est la plus importante. Une masse flottante d'individus dépossédés et sans travail se transforme en petits propriétaires, accède en une génération à un certain seuil de sécurité et de respectabilité ${ }^{29}$. "Si l'ascension sociale la plus évidente concerne l'accession à la terre roturière, la concession des seigneuries, découpées à même la forêt, permit aussi, à un échelon supérieur, à certains individus dont le mérite ne faisait pas de doute aux yeux des autorités coloniales, d'obtenir gratuitement une ou plusieurs seigneuries. Bien que ces seigneurs d'origine modeste aient toujours représenté une minorité parmi les propriétaires seigneuriaux et que leurs fiefs aient le plus souvent eut une superficie proportionnelle à leur condition sociale originelle, on assiste tout de même à des ascensions surprenantes et, surtout, très rapides ${ }^{30}$.

Des pionniers, arrivés aux premières heures de la colonie - tel Pierre Boucher (1622-1717) qui, d'interprète et fils de paysans, devint gouverneur

27. Dans le seul gouvernement de Québec, 62 individus issus des strates inférieures de la roture accèdent, sous le régime français, à la propriété seigneuriale : GRENIER, Benoît, "Devenir seigneur en Nouvelle-France : Propriété seigneuriale et mobilité sociale dans le gouvernement de Québec sous le régime français ", mémoire de maîtrise (histoire), Université Laval (Québec), 2000, 153 folios.

28. Jean-Marie CONSTANT, La noblesse française aux XVI et XVII siècles, Paris, Hachette, 1994 [1985], p. 116.

29. DECHENE, Louise, Habitants et marchands de Montréal au XVII siècle, Montréal, Boréal, 1988 [1974], p. 406.

30. GRENIER, Benoît, " Devenir seigneur... ", op. cit. 
et fut anobli par Louis XIV - bénéficièrent de leur ancienneté et de leur contribution au développement de la Nouvelle-France et, bien qu'issus des milieux les plus modestes (paysannerie, artisanat), ils purent joindre les rangs de la notabilité locale. Avec les familles Couillard et Giffard-Juchereau, la famille Boucher compte parmi les quatre familles nobles du groupe étudié et toutes trois furent anoblies au Canada. Ainsi, parmi les quatre familles nobles en question, seuls les Margane de Lavaltrie l'étaient avant de s'établir en Nouvelle-France. Comme en France, la seigneurie constituait donc au Québec une voie de la promotion sociale qui pouvait aboutir à l'anoblissement. D'autres individus réussirent à acquérir des seigneuries qui, peu peuplées ou inhabitées (et non défrichées ${ }^{31}$ ), leur étaient accessibles financièrement, sans toutefois se voir anoblis. Jean Rioux (1652-1710), immigrant originaire de Ploujean dans le Finistère, qui acquiert la seigneurie de Trois-Pistoles, en 1697, en échange d'une censive sur l'Île d'Orléans, en est un exemple pour le moins intéressant ${ }^{32}$. Ces possibilités s'estomperont avec l'accroissement de la population de la zone seigneuriale, au fil du $\mathrm{XVIII}^{\mathrm{e}}$ siècle, le coût d'achat augmentant considérablement et les concessions devenant plus rares et réservées à des groupes ciblés.

Au moment où la Nouvelle-France passe sous l'autorité de la GrandeBretagne, les seigneuries laurentiennes connaissent certaines transformations causées à la fois par le repli sur leurs terres de certains seigneurs privés de leurs autres champs d'activités, mais surtout en raison de la population qui a crû fortement tout au long du xVIII ${ }^{\mathrm{e}}$ siècle. Les seigneuries, surtout les plus anciennes, deviennent alors densément peuplées, constituant une source de rentabilité non négligeable. Ces seigneuries sont, dès lors, un objet de convoitise pour l'élite britannique, militaire principalement, qui y voit la possibilité de profits, mais aussi la volonté de se doter d'un statut privilégié au sein de la nouvelle colonie. En dépit de la bienveillance des officiers britanniques envers le système et le mode de vie seigneurial, il n'y aura plus, à compter de la Conquête, sauf quelques exceptions, de concessions de seigneuries ${ }^{33}$. Si pendant les premières décennies du régime britannique, le destin du système seigneurial demeure incertain, il sera maintenu jusqu'en 1854, date à laquelle il fut aboli par l'Acte seigneurial. Toutefois, en dépit de l'acquisition par l'élite britannique, marchande et militaire, de nombreuses seigneuries, seule une minorité fut résidante.

31. En Nouvelle-France, une seigneurie qui n'est pas défrichée, tout comme une terre en censive, n'a pratiquement aucune valeur marchande.

32. Archives nationales de Québec à Québec, Acte d'échange entre Charles Denys de Vitré et Jean Rioux et Catherine Leblond, 15 mars 1696 (greffe Louis Chambalon).

33. En 1762, le gouverneur James Murray concédait à deux de ses compatriotes écossais, John Nairne et Malcolm Fraser, les seigneuries de Murray Bay et de Mount Murray, correspondant à l'ancienne seigneurie de La Malbaie qui avait été remise au roi en 1723. $\mathrm{Au}$ total, huit seigneuries furent concédées sous le régime britannique (entre 1762 et 1824). Consulter à ce sujet : BoufFARD, Jean, Traité du domaine, Québec, Le Soleil, 1921, p. 14-18. 
Dans la société rurale laurentienne, historiens et généalogistes rencontrent fréquemment des notables issus de la petite roture et pas seulement parmi le groupe seigneurial; capitaines de milice, professionnels, marchands et hommes politiques sont parfois recrutés au sein de la paysannerie. Dans les seigneuries de la vallée du Saint-Laurent, les exemples d'ascension sociale sont nombreux. Sans vouloir faire écho à l'idée d'une démocratisation par l'intermédiaire du peuplement de colonisation, il n'est toutefois pas abusif de dire que la faiblesse de la population et la disponibilité de la terre ont permis à plusieurs individus modestes d'accéder à des fonctions qu'on aurait pu croire réservées à des catégories sociales plus élevées. Les seigneurs résidants d'origine roturière en constituent un exemple. La présence de ces individus modestes parmi les notables du monde rural laurentien nous incite toutefois à relativiser la portée de la notabilité.

\section{Les seigneurs résidants : la notabilité assurée?}

Traditionnellement, à l'instar de la situation française, on présente le seigneur canadien comme le premier notable de la communauté, surtout s'il réside ${ }^{34}$. "De quelque classe qu'il vienne, mais surtout s'il est anciennement noble, le seigneur est le principal personnage de la communauté rurale. À l'église paroissiale, mort ou vif, il occupe la première place [... ${ }^{35}$. " Après avoir souligné une multitude de manifestations visant à honorer la personne du seigneur, Pierre Goubert note que « le seigneur qui ne réside pas, au moins quelques mois, dans son manoir, obtient rarement les mêmes marques de respect ${ }^{36}$ ». Les seigneurs non résidants ne bénéficient peutêtre pas de la notabilité locale, mais leur absence de la seigneurie étant le plus souvent le fait d'autres occupations, c'est à une autre échelle qu'il faut rechercher leur notabilité. Dans le contexte laurentien, le fait de résider apparaît aussi fondamental pour reconnaître au seigneur une place parmi la notabilité locale. Mais ici comme en France, où les grands seigneurs s'absentent parfois pour se rapprocher de la Cour, l'absence du seigneur dans la vallée du Saint-Laurent n'est pas pour autant synonyme de déchéance sociale : bien au contraire, l'absentéisme est souvent lié à l'exercice de fonctions plus prestigieuses. Inversement, notre étude des seigneurs résidants tend à démontrer que le fait de résider ne suffit pas toujours à assurer au seigneur le statut de premier notable. En effet, l'étude des seigneurs résidants issus de milieux modestes incite à relativiser la notabilité de certains d'entre eux que rien ne distingue vraiment des habitants de leurs seigneuries. Être seigneur dans la vallée du Saint-Laurent suffit-il à se poser en notable et à être reconnu comme tel? Trois indicateurs serviront à illus-

34. MathiEu, Jacques, La Nouvelle-France ..., op. cit., p. 194-195.

35. GouBERT, Pierre, « Les campagnes françaises », Fernand BRAUDEL et Ernest Labrousse (dir.), Histoire économique et sociale de la France, II 1600-1789, Paris, Quadrige/Presses universitaires de France, 1993 [1970], p. 122.

36. Ibid., p. 123. 
trer ce qui nous apparaît comme une difficile intégration à la notabilité pour certaines familles seigneuriales : la faiblesse démographique, le degré d'alphabétisation et la difficile reproduction familiale.

\section{Des seigneuries inhabitées}

Le premier élément qui pousse à nuancer la notabilité des seigneurs résidants tient à la faiblesse démographique qui caractérise ces seigneuries. La seigneurie laurentienne a pris racine dans un contexte de peuplement colonisateur : dans un territoire quasiment inhabité, les premiers seigneurs doivent commencer par installer des censitaires sur leurs fiefs. Plusieurs de ceux que l'on a identifiés comme seigneurs résidants sont arrivés tout au début de la phase colonisatrice et ont fait eux-mêmes œuvre de pionniers. Certes, l'image du seigneur colonisateur, défrichant lui-même ses terres, image qui n'est pas sans rappeler celle des moines-défricheurs du Moyen Âge ${ }^{37}$ et qui a longtemps servi à mythifier le régime seigneurial laurentien, ne rend pas compte de la réalité : il est maintenant évident qu'il s'agissait d'un phénomène exceptionnel. Néanmoins, lorsque l'on s'intéresse au groupe des résidants, le type du seigneur-défricheur réapparaît immanquablement.

La notabilité implique nécessairement une communauté d'individus dont certains occupent une position dominante. Or, la réalité particulière qui existe pendant, à tout le moins, le premier siècle d'existence du régime seigneurial laurentien, est celle d'une mise en place de la population à l'intérieur du corridor que constitue la vallée du Saint-Laurent. Il importe de déterminer, en premier lieu, le moment de l'installation des seigneurs sur leurs domaines seigneuriaux respectifs afin de savoir s'ils précèdent ou non la population. Parmi les dix familles résidantes dans la longue durée auxquelles nous nous intéressons, la quasi-totalité se caractérisa par sa présence pionnière, arrivant avant tout peuplement ou en même temps que les premiers habitants. Ainsi, la présence de familles seigneuriales, pour la plupart roturières et de modestes origines, dans des seigneuries inhabitées, parfois pendant plusieurs décennies, rend inapplicable toute notion de notabilité locale, puisque la seule communauté est alors familiale.

En 1697, Jean Rioux et son épouse, Catherine Leblond, deviennent seigneurs de Trois-Pistoles, seigneurie jusque là inexploitée par ses seigneurs et située dans une région que le peuplement n'a pas encore atteint, en raison de son éloignement. Les nouveaux seigneurs, roturiers, ont accédé à

37. La comparaison de l'évolution historiographique de ces deux phénomènes, malgré des siècles de distance, n'est pas dénuée d'intérêt. Pour le seigneur de la vallée du SaintLaurent, comme pour le moine de la France féodale, l'historiographie, après avoir insisté sur l'action fondamentale de ces deux acteurs dans le processus de défrichement des terres, s'est employée à détruire cette image traditionnelle. Entre ces positions extrémistes, il y a, dans les deux cas, possibilité de nuancer la question. Voir notamment : PICHOT, Daniel, Le Bas-Maine du X $X^{e}$ au XIIt siècle : étude d'une société, Laval, Société d'archéologie et d'histoire de la Mayenne, 1995, p. 72 et suivantes. 
la propriété seigneuriale en échangeant leur terre de l'Île d'Orléans, zone déjà en voie de saturation, contre cette terre seigneuriale sans valeur pour son noble propriétaire, parce que non défrichée et non peuplée ${ }^{38}$. Dès le printemps suivant, la famille Rioux, déjà nombreuse ${ }^{39}$, quitte la région de Québec et se fixe sur sa seigneurie dont elle commence la mise en valeur. Toutefois, les seigneurs Rioux eurent sans doute de la difficulté à y attirer des censitaires, comme en témoigne les aveux et dénombrements déposés au gouverneur en 1723. En effet, vingt-six ans après la venue des Rioux à Trois-Pistoles, ils y sont toujours les seuls habitants, de même que leurs "voisins ", les seigneurs Côté à L'Isle-Verte ${ }^{40}$. Le peuplement finira pourtant par atteindre cette région éloignée de la vallée du Saint-Laurent. À la fin du XVIII ${ }^{\mathrm{e}}$ et au XIX ${ }^{\mathrm{e}}$ siècle, les descendants de Jean Rioux et de Catherine Leblond, pourront peut-être finalement revendiquer une certaine notabilité, probablement redevable autant à l'antériorité de la famille Rioux sur place qu'à son statut seigneurial.

Cet exemple, un peu extrême, en raison de l'isolement de la seigneurie de Trois-Pistoles et du statut social très modeste des Rioux, n'est cependant pas unique parmi les seigneurs résidants de la vallée du Saint-Laurent. À Beauport, Boucherville, Baie-Saint-Antoine ou encore Saint-François-duLac, seigneuries dont la position géographique était beaucoup moins excentrée, les seigneurs précédèrent les censitaires et, malgré l'indéniable position sociale de certains d'entre $e^{41}$, la notabilité dut nécessairement

38. Archives nationales du Québec à Québec, Acte d'échange entre Charles Denys de Vitré et Jean Rioux et Catherine Leblond, 15 mars 1696 (greffe Louis Chambalon).

39. Six enfants vivants composent alors leur famille, un dernier naîtra dans leur seigneurie. 40. MATHIEU, Jacques, LABERGE, Alain (dir.), L'Occupation des terres dans la vallée du SaintLaurent : les aveux et dénombrements 1723-1745, Sillery, Septentrion, 1991 : (aveu et dénombrement de la seigneurie de Trois-Pistoles - 15 février 1723).

41. Le premier seigneur de Boucherville, Pierre Boucher en constitue un exemple évident. La vie de Pierre Boucher a probablement contribué, plus que celle de n'importe quel autre personnage, à amplifier la croyance en une Nouvelle-France se caractérisant par l'ascension sociale. De plus, son rôle comme seigneur de Boucherville est sans doute à l'origine de l'image que les auteurs anciens se sont fait du seigneur-colonisateur. Certes son histoire n'est pas banale et elle lui a valu une statue de bronze sur la façade de l'hôtel du Parlement à Québec, parmi les militaires, les découvreurs et les hommes politiques et religieux qui ont bâti la nation canadienne-française. Né dans le Perche, Pierre Boucher vint en Nouvelle-France en 1635, à l'âge de treize ans, avec ses parents, de simples paysans. Comme plusieurs jeunes garçons de cette époque, il passa quelques années dans les pays d'En-Haut avec les Jésuites; il séjourna parmi les Hurons et apprit les langues amérindiennes; il servit dès lors de truchement. De retour parmi les siens, il entreprit une carrière militaire, devient caporal, puis sergent et combattit contre les incursions iroquoises. Au cours de la décennie 1640, il prit résidence à Trois-Rivières où il fut en charge du magasin de la Compagnie, à titre de commis en chef. Il continua également à combattre les Iroquois à quelques occasions. À compter de ce moment, Pierre Boucher connut une ascension prodigieuse. En 1663, il fut nommé par le gouverneur de Mésy gouverneur des Trois-Rivières, en témoignage de reconnaissance pour sa "Fidélité au service du roi, valeur, expérience, sage conduite au fait des armes... ". En 1661, chargé de plaider devant le roi la cause du Canada, dont il était un des plus anciens habitants, il se rendit en France et pendant cette ambassade, il fut anobli par Louis XIV. 
attendre le peuplement. De plus, même lorsque la population de ces seigneuries aura augmenté, d'autres indices incitent à questionner encore la notabilité seigneuriale de certaines familles résidantes. L'alphabétisation en est une.

\section{Des seigneurs analphabètes ${ }^{42}$ ?}

L'accès à l'instruction (même sommaire) permet parfois à des ruraux de se distinguer de leurs semblables pour occuper des fonctions privilégiées au sein de la collectivité. Dans l'introduction de son ouvrage portant sur les notables du Trégor, Christian Kermoal insiste d'ailleurs sur le critère de l'accès à la connaissance pour définir les élites qui dominent les communautés locales ${ }^{43}$. À l'opposé, l'absence d'un seuil minimal d'instruction chez certains seigneurs résidants amène à nuancer le sens de la notabilité qu'on leur confère habituellement.

On trouve, dans la vallée du Saint-Laurent, des seigneurs et des enfants de seigneurs incapables d'apposer leurs noms à un acte, notarié ou paroissial. Ce phénomène se concentre dans quatre des dix seigneuries de notre étude, toutes propriétés de seigneurs roturiers, parmi les plus modestes du groupe seigneurial. C'est dans les familles seigneuriales de Trois-Pistoles et de L'Isle-Verte que l'incapacité à signer est la plus évidente. À TroisPistoles, au total, sur six générations, dix-sept membres de la famille seigneuriale sont incapables de signer, contre onze qui signent. La proportion d'analphabètes est encore plus saisissante à L'Isle-Verte où, là aussi, sur six générations, dix-neuf fils et filles de seigneurs déclarent ne savoir signer, alors que seulement sept signent au bas des actes notariés. Dans ces deux cas, certaines familles ou générations sont complètement analphabètes, aucun des enfants ne sachant signer, pas même le futur seigneur. Pour certains, la situation apparaît critique à la veille du XIX ${ }^{\mathrm{e}}$ siècle : aucun des six enfants du seigneur Étienne Rioux (1750-1818), pas plus que les huit enfants du seigneur voisin, Jean-Baptiste Côté (1756-1831), ne furent en mesure d'apposer leur signature à leur contrat de mariage. C'est également chez les Côté et les Rioux que se retrouvent les seigneurs incapables de signer. Si à Grondines où à la Baie Saint-Antoine, deux autres seigneuries possédées par des familles seigneuriales d'origine paysanne, à certaines géné-

42. La définition du concept d'alphabétisation par les chercheurs varie grandement et peut se diviser en de nombreux degrés. Nous sommes conscient que la capacité à signer d'un individu ne constitue pas la certitude que cette personne sache écrire; cela veut tout au plus dire qu'il sait signer son nom. Néanmoins, à défaut d'autres pistes, cela demeure le signe tangible d'une capacité toute relative à se servir d'une plume : "En s'en tenant au seul fait de pouvoir ou non signer, on a pu ainsi mesurer l'évolution dans le temps des taux d'alphabétisation, et leurs contrastes entre les régions, les catégories sociales et les sexes ": GRosPERrin, Bernard, "Alphabétisation ", BELY, Lucien (dir.), Dictionnaire de l'Ancien Régime, Paris, Presses universitaires de France, 2002 [1996], p. 50-52.

43. Kermoal, Christian, Les Notables du Trégor. Éveil à la culture politique et évolution dans les paroisses rurales, Rennes, Presses universitaires de Rennes, 2002, p. 14. 
rations, quelques-uns des enfants ne savent signer, le futur seigneur, lui, est au moins capable d'écrire son nom, même si c'est avec difficulté comme en témoigne l'écriture maladroite et barbouillée de certains. Dans le cas des seigneuries de Trois-Pistoles et de L'Isle-Verte, on observe deux générations successives où le seigneur est incapable de signer, ce qui témoigne vraisemblablement de l'absence de toute instruction : après deux seigneurs dotés d'un minimum d'instruction, succèdent deux autres seigneurs incapables de signer, puis, à la fin de la lignée seigneuriale, on retrouve un dernier seigneur à nouveau capable d'écrire son nom et sans doute davantage. Celui-ci témoigne certainement de l'amélioration du système scolaire au cours du XIX ${ }^{\mathrm{e}}$ siècle.

L'éloignement peut sans doute contribuer à expliquer l'analphabétisme plus important dans certaines seigneuries. Ainsi, Trois-Pistoles et L'IsleVerte se situent à l'extrémité orientale de l'œcoumène de la vallée du SaintLaurent. Ces régions furent longtemps isolées, reliées à Québec par voie fluviale exclusivement, et les écoles y arrivèrent tardivement ${ }^{44}$. Mais ce sont aussi deux familles dont les caractéristiques se rapprochent bien souvent davantage de la paysannerie dont elles sont issues que d'un statut social véritablement seigneurial. Les Rioux et les Côté sont roturiers, ils ne sont nullement intégrés à l'élite coloniale et sont fort isolés dans leurs seigneuries éloignées. L'analyse des alliances matrimoniales nous permettrait également de constater ces liens étroits à la collectivité à laquelle ils appartiennent et l'absence d'homogamie ${ }^{45}$.

D'autres familles sont beaucoup mieux positionnées socialement et géographiquement; en témoigne la capacité à signer d'une partie au moins de leurs membres. Ainsi, chez les Boucher, dont la seigneurie de Boucherville se situe sur la rive sud de l'île de Montréal, eux aussi d'extraction modeste, mais anoblis à la première génération canadienne, la totalité des enfants, sur cinq générations, savent signer. Quant aux trois autres familles nobles, à Beauport, à la Rivière-du-Sud et à Lavaltrie, la situation est similaire; tous ceux pour lesquels nous avons l'information s'avèrent capables d'apposer leur signature ${ }^{46}$. De manière générale, malgré certaines familles plus largement analphabètes, les fils et filles de seigneurs semblent majoritairement, de par leur capacité à signer, alphabétisés et, à tout le moins, modestement scolarisés. Néanmoins, dans certains cas, le seigneur se voit très certainement concurrencé par d'autres notables plus instruits et peut difficilement assurer à ses enfants un avenir digne de leur rang.

44. Laberge, Alain, Mathieu, Jacques, "L'expansion de l'œcoumène ", Courville, Serge (dir.), Atlas historique du Québec. Population et territoire, Québec, Presses de l'Université Laval, 1996, p. 45-54.

45. Les renseignements relatifs aux familles Rioux et Côté sont issus de nos travaux de doctorat actuellement en cours.

46. La capacité à signer à été vérifiée systématiquement pour l'ensemble des contrats de mariage disponibles dans chacune des familles seigneuriales pour toute la période où elles furent respectivement propriétaires des fiefs concernés. 


\section{La difficile reproduction familiale des seigneurs résidants}

L'analyse des fonctions ou professions exercées par les membres de la famille seigneuriale, observées par l'intermédiaire des recensements et des actes notariés, soulève également des questions quant à la notabilité de ces individus. On constate que les membres des familles seigneuriales roturières déclarent souvent des fonctions ou des professions qui ne les distinguent nullement des censitaires. On retrouve des fils de seigneurs qui se déclarent cultivateurs ou agriculteurs; ils sont à nouveau issus des familles seigneuriales de L'Isle-Verte et de Trois-Pistoles. On cherchera longtemps le seigneur Jean-Baptiste Côté au recensement de 1851, mais on trouvera un grand nombre de Baptiste Côté qui se déclarent cultivateurs ${ }^{47}$. C'est encore le constat de familles au statut fort précaire qui s'impose. Évidemment, peut-être sont-ils des agriculteurs prospères - ce qui n'a pu être vérifié à ce jour - mais dans les documents, aucune déclaration de fonction ni appellation attestant de leur honorabilité ne les distingue du commun des habitants de leur seigneurie. On doit tenir compte d'une autre donnée qui n'est pas dénuée de sens : l'absence de toute mention de profession ou de fonction chez un grand nombre de fils de seigneurs roturiers; ce sont $36 \%$ d'entre eux qui se trouvent dans ce cas. Pour chacun de ces individus, un ou plusieurs documents les présentent par leur nom, sans plus. Même en supposant que certains notaires soient avares de détails, ce qui est assez peu fréquent lorsqu'il s'agit de familles éminentes, cela constitue le plus important contingent d'individus. Ces fils de familles seigneuriales n'ont probablement aucune fonction les distinguant de la masse paysanne, à laquelle ils semblent assimilés. Dans la mesure où, dans cette société encore fortement hiérarchisée, on ne manque pas de souligner les titres et les fonctions honorifiques, l'absence de désignation professionnelle semble attester qu'ils vivent du travail de la terre et qu'on pourrait sans doute les additionner à ceux qui se déclarent d'emblée cultivateurs, voire même journaliers. Cette assimilation à la communauté paysanne de leur seigneurie ne surprend guère, des phénomènes comparables ont d'ailleurs été soulignés en d'autres régions. À propos de la petite noblesse bretonne, Michel Nassiet écrit :

"Les conditions de vie des familles pauvres [de la noblesse bretonne] [...] : un logis sans signes distinctifs, des vêtements ordinaires... des alliances matrimoniales et une convivialité réduites, de plus en plus, aux voisins paysans. Tout cela a éliminé, dans les familles de noblesse pauvre, la plupart des manifestations symboliques de la noblesse. Après plusieurs générations dans ce style de vie, des lignées ont vu se perdre dans la mémoire de leurs voisins le souvenir de leur différence originelle ${ }^{48}$."

47. Archives nationales du Québec à Québec, Recensement 1851 (Canada-Est), 4M003429, 3430 et 3431 (comté de Rimouski).

48. NASSIET, Michel, Noblesse et pauvreté. La petite noblesse en Bretagne XVe-XVII ${ }^{e}$ siècle, Société d'histoire et d'archéologie de Bretagne, 1997, p. 377. 
En substituant la seigneurie canadienne à la noblesse bretonne, ces lignes pourraient tout aussi bien décrire les seigneurs résidants de la vallée du Saint-Laurent que nous venons d'évoquer.

En nous questionnant sur la signification de la notabilité pour des seigneurs résidants issus de la petite roture et qui sont, à plusieurs points de vue, identifiables à la population paysanne de leurs seigneuries, il est apparu que le seigneur peut ne pas être le notable attendu. Toutefois, il ne faut pas perdre de vue qu'il peut difficilement ne pas l'être aux yeux des habitants de sa seigneurie qui paient les rentes seigneuriales et ont très certainement conscience de son statut distinctif. Avec l'accroissement de la population dans la zone seigneuriale de la vallée du Saint-Laurent (la plus anciennement peuplée), les seigneurs résidants, même les plus modestes, pourront espérer des revenus appréciables de leurs fiefs. La notabilité des familles seigneuriales, dans ce contexte, pourra connaître un renforcement et se prolonger bien au-delà de l'abolition du système seigneurial. Dans la seigneurie de Trois-Pistoles, au cours de la seconde moitié du Xxe siècle, la descendante du dernier seigneur Rioux continuait à être désignée par les habitants des lieux comme " la seigneuresse " et ce, même si, officiellement, le régime seigneurial n'était plus depuis un siècle.

Cette réflexion autour de la question de la notabilité rurale laurentienne et de la difficile intégration de certaines familles seigneuriales résidantes à l'élite locale, voire de leur assimilation à la population paysanne, nous a conduit à insister sur les familles dont la reproduction familiale se caractérise davantage par l'échec que par la réussite. Cependant, sans remettre en question la notabilité des seigneurs laurentiens, dans le contexte d'une historiographie québécoise qui, depuis les années 1970-1980, propose une vision sévère du système seigneurial, insistant sur la nature contraignante de cette institution, il nous apparaît opportun de souligner que ces exemples, puisés parmi les seigneurs résidants, semblent témoigner d'une intégration des seigneurs à la communauté locale plutôt que d'attitudes oppressives.

En poursuivant notre étude des seigneurs résidants de la vallée du SaintLaurent, nous chercherons, entre autres, à préciser la place occupée par ceux-ci dans l'émergence et l'évolution de la notabilité locale. Nous verrons, dans quelle mesure les membres de la famille ont vu leur position se maintenir ou se renforcer au fil du temps, ou si, au contraire, la transmission du patrimoine seigneurial a fait en sorte que des fils et des filles de la famille seigneuriale ont dû déroger à leurs origines seigneuriales et se voir intégrés à la communauté, sans égard au statut originel de leur famille, les distinguant de la masse paysanne. Est-ce que, à l'instar des familles paysannes incapables d'assurer à leur progéniture une reproduction à l'identique et devant migrer vers d'autres lieux, des membres des familles seigneuriales furent intégrés au monde des paysans et des journaliers ${ }^{49}$ ? Pour

49. Concernant ces questions pour la paysannerie québécoise, un ouvrage incontournable : Bouchard, Gérard, Quelques arpents d'Amérique. Population, économie, famille au Saguenay 1838-1971, Montréal, Boréal, 1996, 635 p. 
une partie d'entre eux, ceux dont nous avons traité dans ce texte, nous croyons que tel fut le cas. En contrepartie, pour d'autres familles résidantes, celles appartenant à la noblesse principalement, la notabilité locale ou régionale pourra constituer une porte de sortie aux exclus du titre seigneurial, faisant apparaître une dichotomie au sein du sous-groupe des seigneurs résidants de la vallée du Saint-Laurent.

\section{RESUME}

Pendant plus de deux siècles, la vallée du Saint-Laurent (Québec) a vécu sous le système seigneurial, implanté au début du régime français, maintenu par les Britanniques, puis aboli en 1854. En nous intéressant aux seigneuries habitées par la famille seigneuriale, dans le contexte colonial laurentien, nous illustrerons les spécificités de la notabilité rurale du Québec préindustriel. Les rapports entre notables locaux et famille seigneuriale dans les seigneuries laurentiennes permettent d'observer une mobilité sociale qui introduit des individus d'humbles origines au sein des propriétaires de seigneuries, notamment parmi le groupe des seigneurs résidants. L'observation de certaines familles seigneuriales résidantes incite à relativiser leur statut de notables.

\section{ABSTRACT}

For more than two centuries, Saint-Lawrence Valley (Quebec) lived under seigneurial system, established at the beginning of French regime, maintained by the British, and abolished in 1854. By studying the seigneuries inhabited by seigneurial families, this paper seeks to illustrate the specificities of rural notability in preindustrial Quebec, in a period of settlement. Relations between local notables and seigneurial families provide an opportunity to examine the social mobility which insert many commoners among Canadian seigneurs, especially in the groupe of the seigneurs who reside. The analysis of some seigneurial families tends to question their notable status. 
